\title{
Advanced Primary Lymphoma of Oral Cavity: Report of a Case
}

\author{
Somayeh Alirezaei', Maryam Baharvand2, Bita Tavakoli1, Soodeh Sarikhani' ${ }^{3}$, \\ Ahmad R. Mafi ${ }^{*}$ \\ ${ }^{1}$ Department of Oral and Maxillofacial Medicine, Dental School, Islamic Azad University, Tehran, Iran \\ ${ }^{2}$ Department of Oral and Maxillofacial Medicine, Dental School, Shahid Beheshti University of Medical Sciences, \\ Tehran, Iran \\ ${ }^{3}$ Department of Oral and Maxillofacial Radiology, Golestan University of Medical Sciences, Gorgan, Iran \\ ${ }^{4}$ Department of Radiation Oncology, Imam Hossein Hospital, Shahid Beheshti University of Medical Sciences, \\ Tehran, Iran \\ Email: ahmadrmafi@gmail.com
}

Received 1 January 2014; revised 3 February 2014; accepted 12 February 2014

Copyright (C) 2014 by authors and Scientific Research Publishing Inc.

This work is licensed under the Creative Commons Attribution International License (CC BY).

http://creativecommons.org/licenses/by/4.0/

(c) (i) Open Access

\begin{abstract}
Usually the oral manifestations of NHL are secondary to a more widespread involvement throughout the body, however, it can rarely present as a primary lesion in the oral cavity, having $0.1 \%$ $0.2 \%$ prevalence. In this paper we report a case of highly proliferative oral non-Hodgkin lymphoma which presented with very mild symptoms, but had a sudden and significant progression in less than two weeks with involvement of all four quadrants of the oral cavity.
\end{abstract}

\section{Keywords}

Lymphoma; Intra Oral Swelling; Four Quadrant

\section{Introduction}

Lymphomas are malignant neoplasms of the lymphocyte cell lines. Histologically they are characterized by the presence or absence of Reed-Sternberg cells. Reed-Sternberg cells are pathognomic of Hodgkin's disease, and all other lymphoid neoplasms that lack these cells are characterized as non-Hodgkin's Lymphomas (NHL). They mainly involve lymph nodes, spleen and other non-hemopoietic tissues, with painless enlargement of lymph nodes being the most common presenting symptom. However, an important feature of NHL which distinguishes it from Hodgkin's disease is that NHL can arise in an extranodal site, either as a primary or occult lesion [1] [2].

Corresponding author. 
Although the predominant sites of lymphoma occurrence are lymph nodes, it also occurs in other organs. Most NHLs arise in lymph nodes or other lymphatic tissues such as the spleen, Waldeyer's ring and thymus [2]. Involvement of extranodal organs is a common finding after staging investigation, however, about $25 \%$ - $40 \%$ of NHL patients present with a primary extranodal lymphoma, and common presenting sites include gastrointestinal tract, pharynx, thyroid, lung, central nervous system, orbit, salivary glands and skin [3] [4]. In head and neck, lymphomas are the most common neoplasms second to squamous cell carcinoma, and make up $5 \%$ of all malignancies of head and neck. Moreover, the head and neck is the second most common region for the extra-nodal lymphomas after that of gastrointestinal Tract [1] [2].

Usually the oral manifestations of NHL are secondary to a more widespread involvement throughout the body, however, it can rarely present as a primary lesion in the oral cavity, having $0.1 \%-0.2 \%$ prevalence [4] [5]. Waldeyer's ring is the most common site of lymphomas involving the oral region. Though primary intraoral lesions in NHL are uncommon, it is important to be aware of them, since intraoral manifestations are the presenting symptom in these patients. In this study, we report a case of maxillofacial and intraoral lymphoma with rapid growth and bony destruction.

\section{Case Report}

A 32-year-old woman was seen at our center complaining of severe pain and swelling in her mouth that had been developed in the last two weeks. On history taking, she mentioned that she visited her dentist for a small lesion she felt in her posterior tooth of left mandible. She was not able to remember the time when the lesion appeared, but she mentioned that the mild pain and discomfort had started a couple of days before the day she visited her dentist. With the diagnosis of infection, the tooth was extracted by the dentist and she was prescribed antibiotics. After a few days, her oral cavity started to swell, so that she developed mild to moderate dysphagia. The pain and swelling worsened dramatically and she was referred to the oral medicine department for further investigation. Extraoral examination revealed asymmetry and swelling of the left side of the face. Intra-oral exam showed a firm mass on the right side of the maxillary ridge (buccal and palatal), $4-5 \mathrm{~cm}$ in diameter and also a $4-5 \mathrm{~cm}$ firm mass on mandibular ridge on the same side that had resulted in gingival erosion, with bosselated surface and pink color (Figure 1).

The lesion was also associated with a bulky cervical mass growing on the right cervical region, starting from the submaxillary region up to the supra-clavicular region. Examination of the rest of the body was normal, and
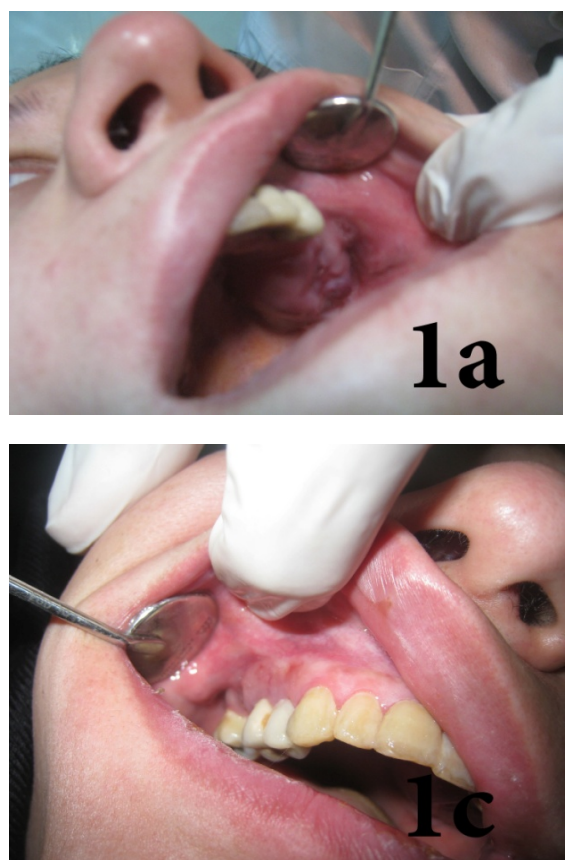
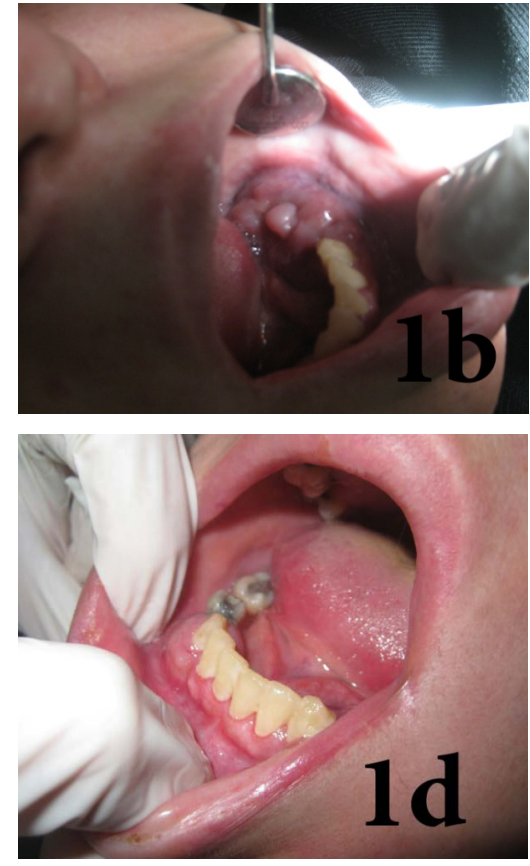

Figure 1. Patient's mouth on clinical examination. (a) Left upper quadrant of the mouth; (b) Left lower quadrant of the mouth; (c) Right upper quadrant of the mouth; (d) Left lower quadrant of the mouth. 
there was no fever or night sweats. She only mentioned a slight weight loss in the past two week because of improper eating due to dysphagia and pain.

Head and neck computed tomography scan showed a $48 \times 36 \mathrm{~mm}$ soft tissue mass with bony destruction in the body of left mandible, with extension to alveolar ridge and inner side of mandible; a tumoral lesion in left maxilla with extension to left maxillary sinus causing destruction the floor of left antrum; and multiple lymphadenopatheis in submandibular and jugulodigastric chains bilaterally (Figures 2 and 3).

An incisional biopsy was performed. Microscopic, immunohistochemical and molecular features of the reported case confirmed the diagnosis of primary diffuse large B-cell Lymphoma of the oral cavity (Figures 4(a)-(c)). according to the Revised European-American Classification of Lymphoid Neoplasms/World Health Organization classification of lymphoid neoplasms (REAL/WHO).

Staging work-up was performed. There were no signs of the disease elsewhere in the body. Computed tomography and MRI scans of the chest, abdomen and pelvis, as well as bone marrow biopsy did not show any abnormalities. Laboratory tests including renal and liver function tests were within normal limits and the patient was HIV negative. The result of Ki67 returned as $>90 \%$.

The patient was referred for chemotherapy. After two cycles of R-CHOP regimen, the oral lesions regressed significantly and the patient became symptom free (Figure 5).

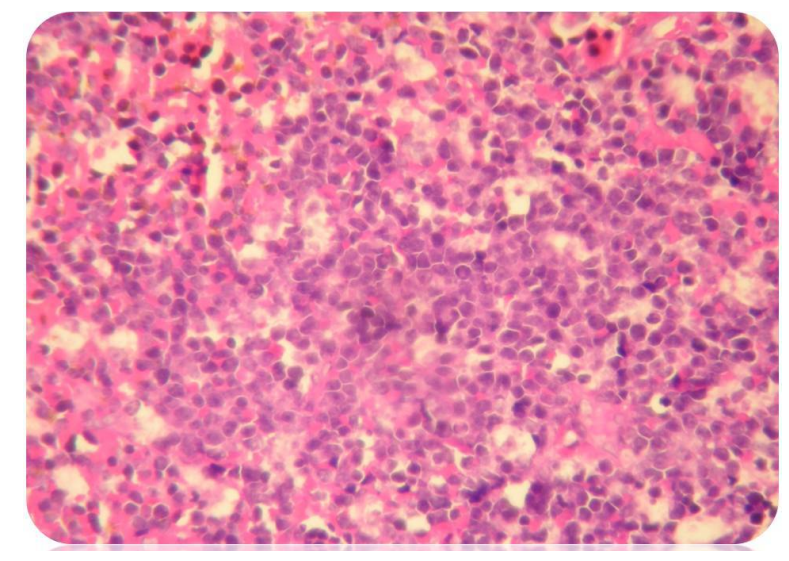

(a)

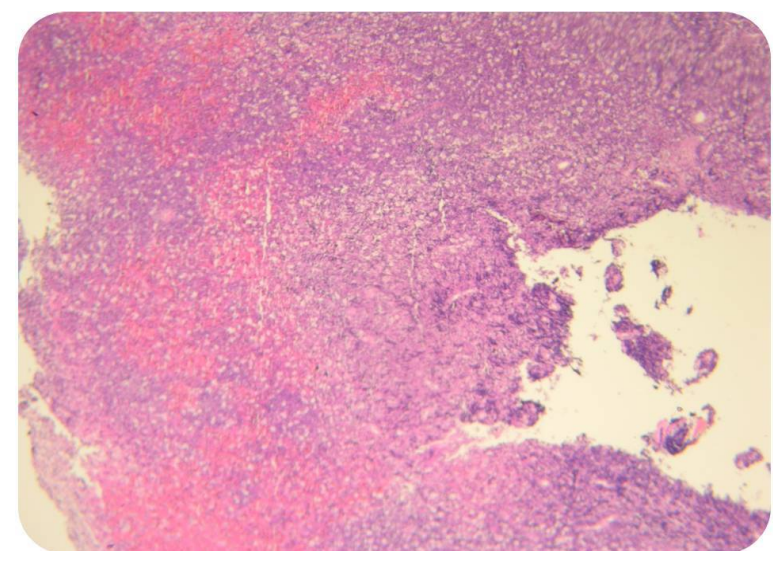

(b)

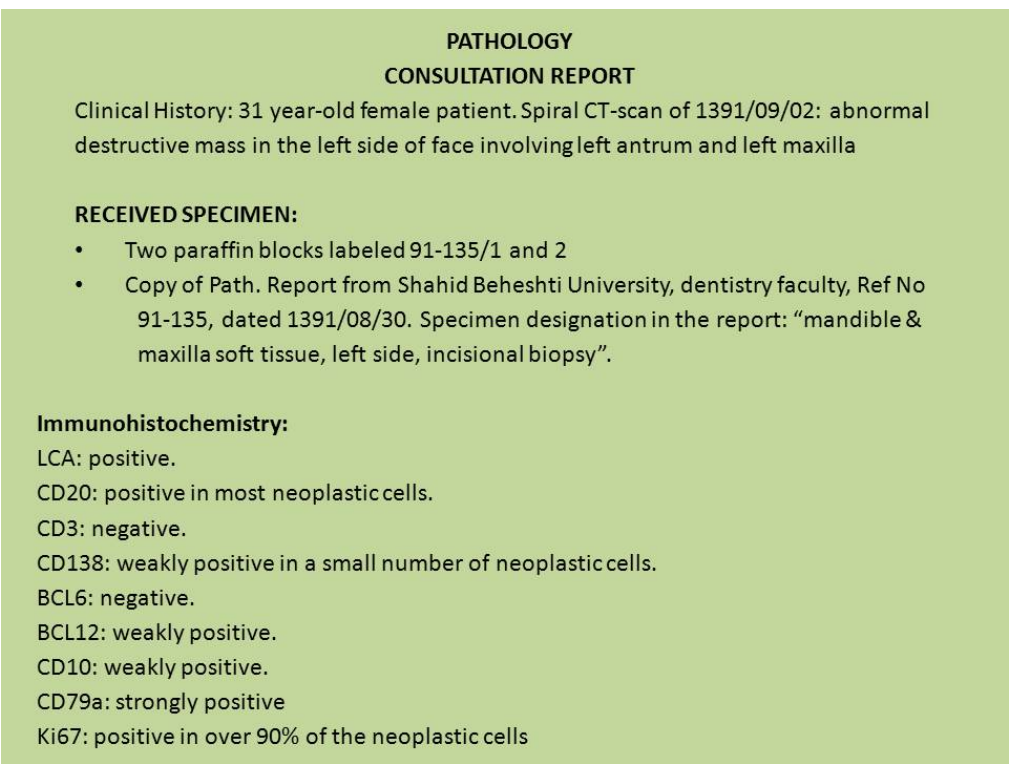

(c)

Figure 2. (a) Histological examination Specimen biopsied showing the non hodgkins lymphoma diffuse large Bcell typeH \& E stain. Magnification ×400; (b) Necrotic foci in diffuse large B cell lymphoma; (c) IHC REPORT. 


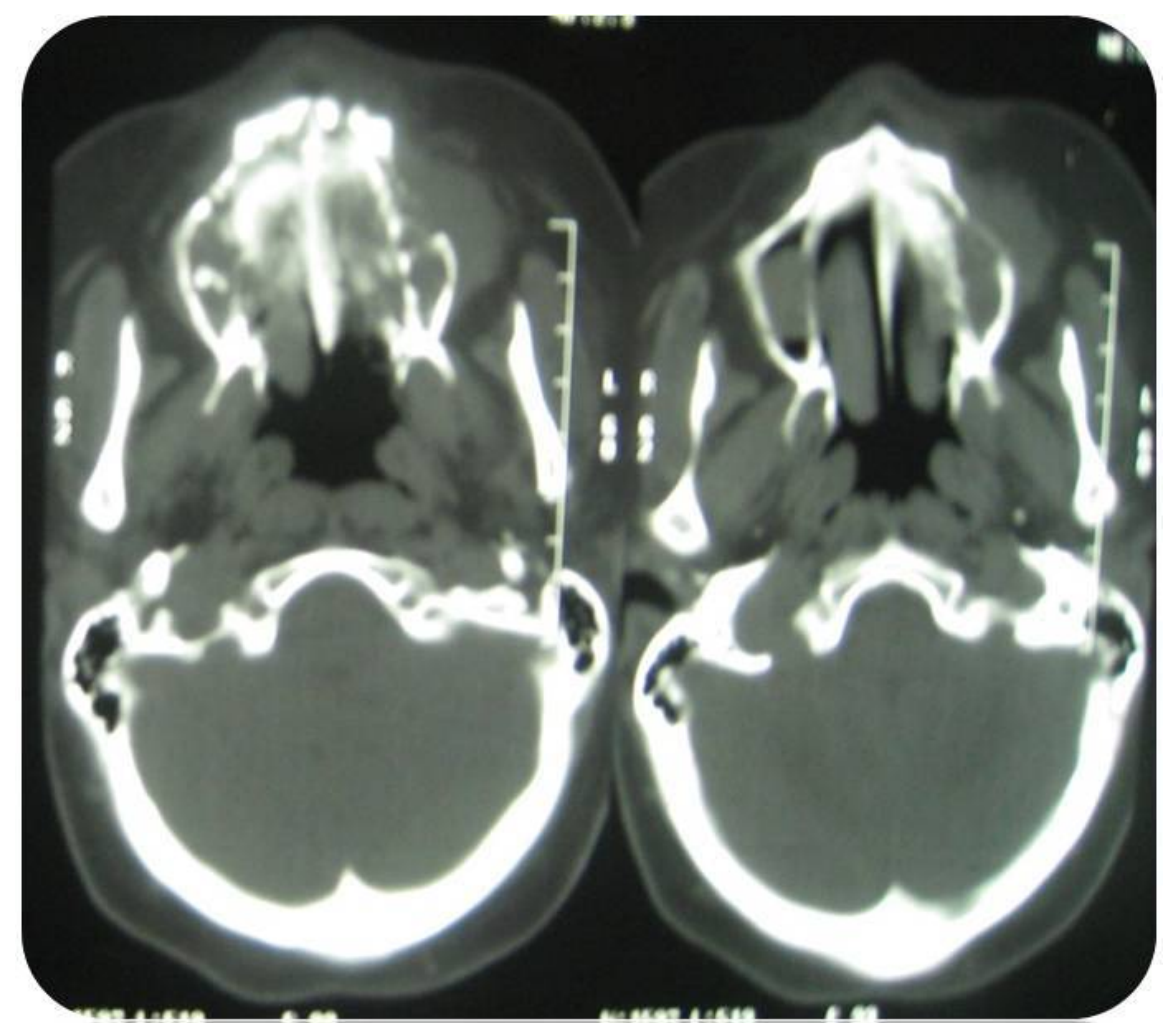

Figure 3. An axial CT image with soft tissue algorithm revealing irregular resorbtion of the left maxilla.

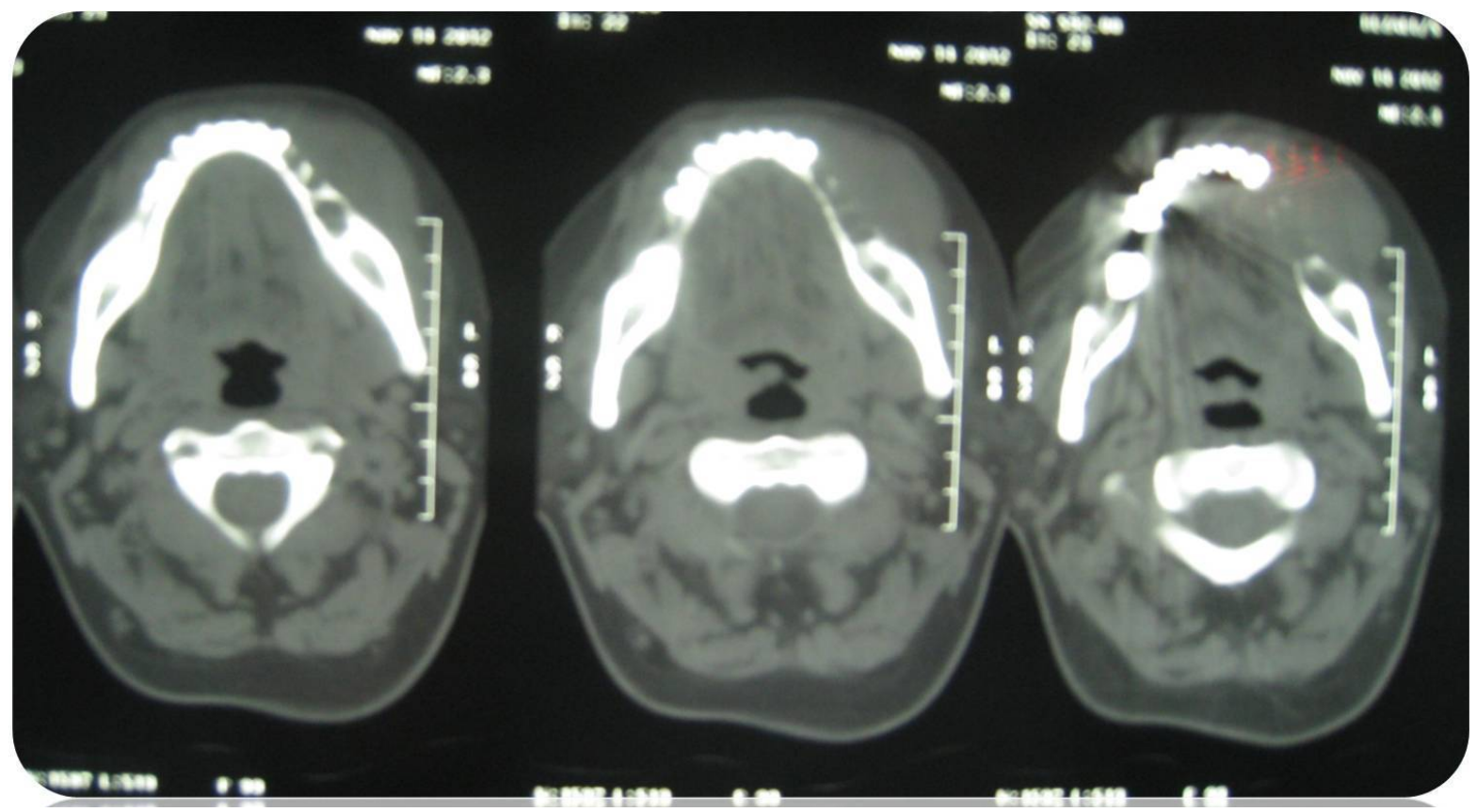

Figure 4. An axial CT image with soft tissue algorithm revealing irregular resorbtion of the left mandibule.

She has recently completed her chemotherapy and radiotherapy treatments, and at present she is still symptom free with no signs of recurrence/unresponsiveness. Regular follow-up visits have been arranged for her at our center. 


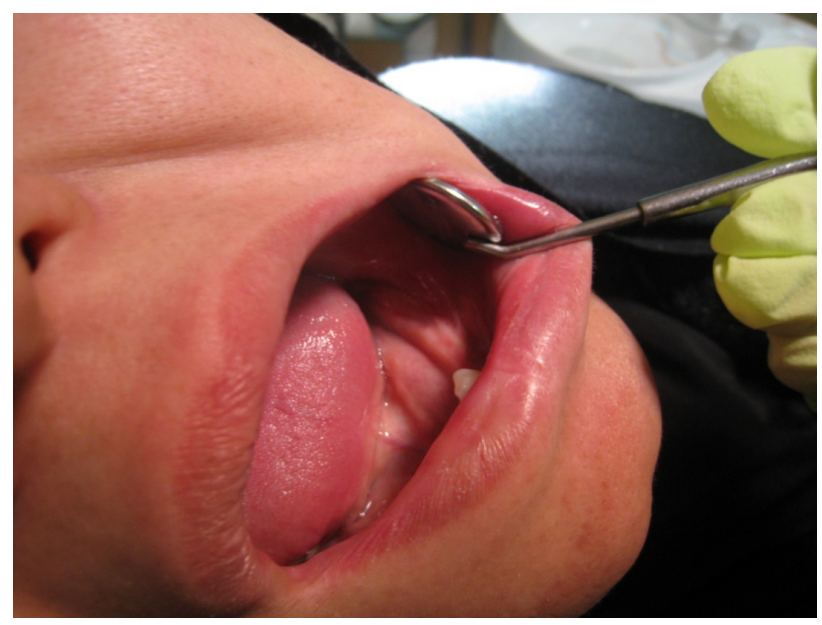

Figure 5. Patient's mouth after first cycle of chemotherapy (compare with Figure 1(b)).

\section{Discussion}

The incidence of head and neck NHL has increased in the last few years. The incidence increases with age and generally older adults are involved. Furthermore, the incidence of oral lesions as the first presentation of a systemic lymphoma also seems to be increasing. Study of Vander Waal et al., on 40 of primary extranodal NHL of the oral cavity, showed that the mean age of developing the disease was 59 years (3 - 88 years) and male gender was more commonly involved. In their study, in $66 \%$ of the cases, the lesion arose from soft tissues, and $77 \%$ of these were seen in the upper jaw [6].

The most common site involved in oral cavity was also showed to be palate and gingiva. In, Shah GH et al. study on 15 cases of isolated primary extranodal lymphoma of the oral cavity; they reported the upper gingivobuccal complex as the most common site of involvement [7]. Guevara-Canales et al. in a systematic review of 15 studies and a total 714 patients, reported that the most frequent intra-oral location was gingiva, followed by palate [1]. Many studies have shown that primary oral lymphomas present with a soft to firm soft tissue mass which is usually non-tender and may or may not be ulcerated. This lesion can mimic periodontal or apical abscesses [8] [9]. Bony lesions, however, often present with low grade pain which can mimic a toothache making the diagnosis difficult. Radiographically, these bony lesions appear very similar to osteomyelitis or other malignancies creating even more difficulty in diagnosis [1]. Studies have also shown that jaw involvement by NHL is rare, but among jaw lesions, maxilla is more frequently involved than mandible.

In our case, the patient's main complaint was a rather severe pain which stopped her from proper eating, which could be due to diffuse involvement of soft tissue and bones in all four quadrants of the oral cavity. This widespread involvement of the oral cavity is rather uncommon, and what makes this case interesting is the fact that she only had a slight pain in one of her teeth, with no other sign and symptoms, when she first went to see her dentist. It is interesting how such an aggressive tumor (with Ki67 > 90\%) which caused swelling of all four quadrants of the oral cavity in less than two weeks, did not have any symptoms just two weeks before the diagnosis, apart from a mild ache in one of the teeth.

\section{Conclusion}

Although rare, NHL can involve facial bones with only mild and non-characteristic symptoms. The diagnosis of oral lymphomas may be challenging because frequently there is a low index of clinical suspicion, leading to misdiagnosis or delayed treatment. Therefore, it is important that health care professionals be more alert about this malignancy, and perform a thorough physical examination, supported by proper radiological and histopathological investigations in order to identify the disease at an earlier stage.

\section{References}

[1] Canales, J.O., Vadillo, R., de Faria, P.E., Contreras, S.J., Leite, F.P. and Chaves, M.G. (2011) Systematic Review of 
Lymphoma in Oral Cavity and Maxillofacial Region. Acta Odontológica Latinoamericana, 24, 245-250.

[2] Essadi, I., Ismaili, N., Tazi, E., Elmajjaoui, S., Saidi, A., Ichou, M., et al. (2008) Primary Lymphoma of the Head and Neck: Two Case Reports and Review of the Literature. Cases Journal, 1, 426. http://dx.doi.org/10.1186/1757-1626-1-426

[3] Krol, A.D., le Cessie, S., Snijder, S., Kluin-Nelemans, J.C., Kluin, P.M. and Noordijk, E.M. (2003) Primary Extranodal Non-Hodgkin's Lymphoma (NHL): The Impact of Alternative Definitions Tested in the Comprehensive Cancer Centre West Population-Based NHL Registry. Annals of Oncology, 14, 131-139. http://dx.doi.org/10.1093/annonc/mdg004

[4] Ramani, P., Ahmed, S. and Janaki, V.R. (2004) Primary Extranodal Non-Hodgkin’s Lymphoma of the Oral Cavity. Indian Journal of Dermatology, Venereology and Leprology, 70, 172-174.

[5] Epstein, J.B., Epstein, J.D., Le, N.D. and Gorsky, M. (2001) Characteristics of Oral and Paraoral Malignant Lymphoma: A Population-Based Review of 361 Cases. Oral Surgery, Oral Medicine, Oral Pathology, Oral Radiology, and Endodontology, 92, 519-525. http://dx.doi.org/10.1067/moe.2001.116062

[6] Van der Waal, R.I., Huijgens, P.C., van der Valk, P. and van der Waal, I. (2005) Characteristics of 40 Primary Extranodal Non-Hodgkin's Lymphomas of the Oral Cavity in Perspective of the New WHO Classification and the International Prognostic Index. International Journal of Oral and Maxillofacial Surgery, 34, 391-395. http://dx.doi.org/10.1016/j.ijom.2004.08.009

[7] Shah, G.H., Panwar, S.K., Chaturvedi, P.P. and Kane, S.N. (2011) Isolated Primary Extranodal Lymphoma of the Oral Cavity: A Series of 15 Cases and Review of Literature from a Tertiary Care Cancer Centre in India. Indian Journal of Medical and Paediatric Oncology, 32, 76-81. http://dx.doi.org/10.4103/0971-5851.89776

[8] Bhattacharyya, I., Chehal, H.K., Cohen, D.M. and Al-Quran, S.Z. (2010) Primary Diffuse Large B-Cell Lymphoma of the Oral Cavity: Germinal Center Classification. Head and Neck Pathology, 4, 181-191. http://dx.doi.org/10.1007/s12105-010-0184-4

[9] Jayakrishnan, R., Thomas, G., Kumar, A., Nair, R.A. and Mathews, S. (2011) Non-Hodgkin's Lymphoma of the Hard Palate. Journal of the Indian Medical Association, 109, 755-756. 